doi:10.17659/01.2021.0042

Journal of Case Reports 2021;11(3):160-163

\title{
Possible Role of Intravenous Hyaluronidase Treatment in Coronary Lesion and Hypertension
}

\author{
Andreas Pfützner ${ }^{1,2}$, Daniela Sachsenheimer ${ }^{1}$, Gunther Burgard ${ }^{3}$ \\ ${ }^{1}$ Pfützner Science \& Health Institute, Mainz, Germany; ${ }^{2}$ University for Digital Technologies in Medicine \& Dentistry, Wiltz, \\ Luxembourg; ${ }^{3}$ PharmaACT AG, Berlin, Germany.
}

\section{Corresponding Author:}

Dr Andreas Pfützner

Email: andreas.pfuetzner@pfuetzner-mainz.com

This is an Open Access article distributed under the terms of the Creative Commons Attribution License (creativecommons.org/ licenses/by/3.0).

$\begin{array}{lll}\text { Received } & : & \text { January 20, } 2021 \\ \text { Accepted } & : & \text { May 11, 2021 } \\ \text { Published } & : & \text { July 30, 2021 }\end{array}$

\begin{abstract}
Background: Coronary artery disease is the major cause of death in many countries. It is treated by surgical measures in combination with anticoagulants to protect from thrombosis. In late stage and for patient rejecting the modern surgical procedures or drugs, no further treatment options exist. Case Report: A 60-year-old man suffered from painful peri-arthritis in the right shoulder. He also had arterial hypertension with occasional severe hypertensive crises. Successful treatment of the orthopedic problem by means of a series of ten intravenous high-dose hyaluronidase infusions removed not only the shoulder pain, but had additional beneficial effect on the coronary artery disease and hypertension. A stress-induced ischemia, which was detected by MRI prior to the treatments, disappeared, and the patient remained normotensive without further need for drugs for the next five years. Conclusion: A beneficial impact of intravenous hyaluronidase treatment on atherosclerotic lesions has been reported already 50 years ago and can be explained by the molecular action of the enzyme. However, research on this topic ceased, when stents and other more compelling treatment methods were detected. Appropriately designed clinical studies are required to confirm the value of hyaluronidase infusions as treatment alternative, in particular for late-stage atherosclerosis.
\end{abstract}

Keywords: Anticoagulants, Atherosclerosis, Coronary Artery Disease, Shoulder, Pain, Thrombosis.

\section{Introduction}

Macrovascular disease represents the major cause of mortality in industrialized countries. In Germany, myocardial infarction, stroke, and other consequences of atherosclerosis caused $40 \%$ of all reported deaths in 2012 [1]. Treatments for atherosclerosis may include lifestyle changes, medicines, and medical procedures or surgery. The goals of treatment include relieving symptoms, reducing risk factors in an effort to slow or stop the buildup of plaque, lowering the risk of blood clot forming, widening or bypassing plaque-clogged arteries, and preventing atherosclerosis-related diseases. In severe cases of the disease, when plaques have already been formed and start to impact vascular function and blood flow, surgical procedures are usually applied. Angioplasty with optional placing of a stent can improve blood flow to the heart and relieve chest pain. Coronary artery bypass grafting is used to bypass narrowed coronary arteries by using healthy blood vessels for replacement e.g., veins from the leg. Additionally, carotid endarterectomy may be applied to remove plaque buildup from the carotid arteries in the neck, and to restore blood flow to the brain for stroke prevention. These surgical measures in combination with anticoagulants to protect from thrombosis represent the current state of the art in late-stage atherosclerosis and no further approved treatment option exists at this stage. Therefore, there is need to develop further treatment alternatives in particular for patients 
with late-stage atherosclerosis [2,3]. Next to other indications, intravenous treatment with the enzyme hyaluronidase is approved in Germany for treatment of inflammatory conditions of the connective tissue such as tendovaginitis, arthritis or myofascicular pain syndromes $[4,5]$. Because of the anti-inflammatory effects of hyaluronidase, it has been speculated that the enzyme may also have beneficial effects on other conditions driven by inflammation, such as atherosclerosis.

Here we report on a single case study, where treatment of an orthopedic problem by means of high-dose hyaluronidase infusion resulted in efficient removal of a previously unknown atherosclerotic lesion in the heart and in normalization of blood pressure and vascular elasticity.

\section{Case Report}

The patient was a 60 -year-old man with arterial hypertension (treated with $5 \mathrm{mg}$ bisoprolol/day) and a long-term history of painful peri-arthritis in the right shoulder. To remove the shoulder pain, the treating physician started a series of ten hyaluronidase treatment procedures over a period of three weeks. The employed hyaluronidase product is approved for arthritis treatment in Germany (Hylase-Dessau, Riemser Pharma). Infusions were made within 60 min with 15,000 U of hyaluronidase in physiological sodium chloride solution by means of an infusion pump. This dose is a three times higher dose than normally recommended to enhance anti-inflammatory efficacy. Therefore, the patient gave explicit written informed approval for this experimental treatment and each infusion was monitored with continuous pulse and blood pressure surveillance. All individual treatment procedures were very well tolerated, and no adverse event was reported. During the first infusion, blood pressure decreased within one hour from 140/90 $\mathrm{mmHg}$ to $115 / 80 \mathrm{mmHg}$. The patient decided to stop the antihypertensive medication on the next morning on his own because of low blood pressure values. He remained normotensive without medication for the further remaining treatment and observation period. Pulse wave velocity was $11.6 \mathrm{~m} / \mathrm{sec}$ before the first and $9.5 \mathrm{~m} / \mathrm{sec}$ directly after the last infusion.

In a parallel approach to also investigate the potential reason for the occasional hypertensive crises, a stress MRT was performed at an independent site as part of an annual cardiology check prior to initiation of the hyaluronidase treatment. The stress MRI immediately prior to the first hyaluronidase procedure gave evidence of a sub-endocardial ischemic lesion not known before occurring during the stress phase of the MRI after adenosine application [Fig.1a], which was classified as requiring a stent intervention by the radiologist and the cardiologist. However, when receiving this information, the patient had already passed half of the planned hyaluronidase infusions and felt comfortable. Against medical advice, he decided on his own to finish the treatment and rerun the MRI analysis after several weeks. In the meantime, he did not require any anti-hypertensive medication and did not experience any hypertensive crisis.

In repeated MRI examination six weeks after the last hyaluronidase treatment, there was no further evidence of any ischemia anymore [Fig.1b] and the results were classified as "normal heart
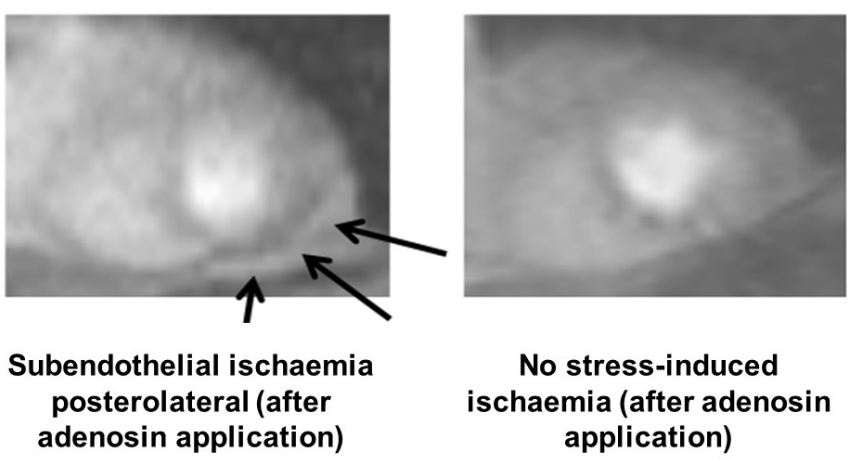

Fig.1: MRI assessments before and after the hyaluronidase infusions (both screen shots made during the stress phase after adenosin application). An endocardial ischemic lesion became visible prior to the hyaluronidase intervention, which had disappeared 10 weeks after the treatment. 
for age in contrast to the previous analysis" by the same investigator. The pain in the shoulder had disappeared and in the course of the following five years, the patient remained normotensive without requiring anti-hypertensive medication. In addition, he reported about a subjective increase in physical fitness, e.g., when doing his usual weekend biking tours, which was also confirmed by his wife.

\section{Discussion}

Proteoglycans and hyaluronan play a key role in the genesis, formation, progression and composition of atherosclerotic plaques. Together with lipids, lipoproteins and calcium, they form complexes that built the morphological skeletal backbone of the atherosclerotic plaque. Hyaluronan (also known as macromolecule of hyaluronic acid, HA) is a large, non-sulfated glycosaminoglycan that is ubiquitously present in the extra-cellular matrix of all vertebrates, with high-molecular weight forms being the physiologically normal isoform. It has been shown that during inflammation and tissue injury, low-molecular-weight fragments are predominantly built, which exert angiogenic and pro-inflammatory effects [4-6]. In consequence, remodeling of the HA content of the glycocalix in favor of high-molecular weight isoforms may be a therapeutic target for treatment of atherosclerosis [7]. Hyaluronan is a dynamic molecule with a high rate of metabolism. In humans, the turnover rate for hyluronan is $5 \mathrm{~g} /$ day of the total $15 \mathrm{~g}$ in the body [8]. The majority of the hyaluronan in the vasculature is incorporated into the endothelial glycocalyx and the extracellular matrix of the underlying tissue [912]. Hyaluronan content and composition of the glycocalix plays a major role in the permeability of the layer. Treatment with hyaluronidase has been shown to increase permeability in post-capillary venules in the rat [10].

It is therefore postulated that treatment of the vasculature with hyaluronidase may result in the rapid formation of a new and less damaged glycocalix in patients with chronic system inflammation. In addition, the enhanced permeability after intravenous administration may allow the enzyme to also penetrate into the smooth muscle cell layer. The hyaluronidase molecules may reach the plaques, where hyaluronan is also present as one of the major skeletal components of the plaque itself [13-16]. After cleaving the solid proteoglycans, the plaque may become more flexible and may better be targeted and dissolved by further self-repair mechanisms within the arterial wall. In animal experiments, exposure of atherosclerotic tissues with plaque lesions to bacterial hyaluronidase resulted in fast and effective degradation of the plaques [18]. In anecdotal clinical cases, it has been demonstrated that atherosclerotic plaques can be reduced or even removed by intravenous administration of high doses of hyaluronidase in patients with coronary heart disease and/or arterial obstructive disease resulting in improved vascular function in late-stage atherosclerosis [18,19]. A beneficial impact of intravenous hyaluronidase treatment on atherosclerotic lesions has been reported already 40-50 years ago [20-22] and can be explained by the molecular action of the enzyme. However, research on this topic ceased, when stents and other seemingly more compelling treatment methods were detected. Based on our experience, we recommend restarting this research and investigate hyaluronidase infusions as treatment alternative, in particular for improvement of late-stage atherosclerosis.

\section{Conclusion}

Intravenous treatment of a joint inflammation with hyaluronidase resulted in additional sustained normalization pressure and elimination of a previously unknown stress induced ischemia in the coronary arteries. Controlled clinical studies are now warranted to verify and confirm the antiatherosclerotic activity of hyaluronidase, when given by intravenous infusion. 
Contributors: AP: manuscript writing, patient management; DS: manuscript editing, patient management; GB: critical inputs into the manuscript. AP will act as a study guarantor. All authors approved the final version of this manuscript and are responsible for all aspects of this study.

Funding: None; Competing interests: None stated.

\section{References}

1. Federal German Bureau of Statistics, Wiesbaden: https://www.destatis.de/DE/Publikationen/Thematisch/ Gesundheit/Todesursachen/Todesursachen.html. Accessed on January $10^{\text {th }}, 2021$.

2. Rydén L, Grant PJ, Anker SD, Berne C, Cosentino $\mathrm{F}$, Danchin N, et al. ESC guidelines on diabetes, prediabetes, and cardiovascular diseases developed in collaboration with the EASD - summary. Task Force on diabetes, pre-diabetes, and cardiovascular diseases of the European Society of Cardiology (ESC); European Association for the Study of Diabetes (EASD). Eur Heart J. 2013;34(39):3035-3087.

3. Brott TG, Halperin JL, Abbara S, J. Michael Bacharach JM, Barr JD, Bush RL, et al. 2011 ASA/ACCF/AHA/ AANN/AANS/ACR/ASNR/CNS/SAIP/SCAI/SIR/ SNIS/SVM/SVS guideline on the management of patients with extracranial carotid and vertebral artery disease: executive summary. Circulation. 2011;124:489532.

4. Perk J, De Backer G, Gohlke H, Graham I, Reiner $\mathrm{Z}$, Verschuren $\mathrm{M}$, et al. European Guidelines on cardiovascular disease prevention in clinical practice (version 2012): The Fifth Joint Task Force of the European Society of Cardiology and Other Societies on Cardiovascular Disease Prevention in Clinical Practice (constituted by representatives of nine societies and by invited experts). Atherosclerosis. 2012; 223:1-68.

5. Jiang D, Liang J, Noble PW. Hyaluronan as an immune regulator in human diseases. Physiol Rev. 2011;91:221264.

6. Stern R, Asari AA, Sugahara KN. Hyaluronan fragments: an information rich system. Eur J Cell Biol. 2006;85:699715.

7. Itano N, Sawai T, Yoshida M, Lenas P, Yamada Y, Imagawa $\mathrm{M}$, et al. Three isoforms of mammalian hyaluronan synthases have distinct enzymatic properties. J Biol Chem. 1999;274:25085-92.

8. Kashima Y, Takahashi M, Shiba Y, Itano N, Izawa A, Koyama J, et al. Crucial role of hyaluronan in neointimal formation after vascular injury. PLoS One. 2013;8(3):e58760.

9. Fraser JR, Laurent TC, Laurent UB. Hyaluronan: its nature, distribution, functions and turnover. J Intern Med. 1997;242:27-33

10. Wheeler-Jones CP, Farrar CE, Pitsillides AA. Targeting hyaluronan of the endothelial glycocalyx for therapeutic intervention. Curr Opin Investig Drugs. 2010;11:9971006.

11. Gao L, Lipowsky HH. Composition of the endothelial glycocalyx and its relation to its thickness and diffusion of small solutes. Microvasc Res. 2010;80:394-401.

12. Henry CB, Duling BR. Permeation of the luminal capillary glycocalyx is determined by hyaluronan. Amer J Physiology. 1999;277:H508-514.

13. Gouverneur M, Spaan JA, Pannekoek H, Fontijn RD, Vink H. Fluid shear stress stimulates incorporation of hyaluronan into endothelial cell glycocalyx. Am J Physiol Heart Circ Physiol. 2006;290:H458-452.

14. Evanko S, Raines EW, Ross R, Gold LI, Wight TN. Proteoglykan distribution in lesions of atherosclerosis depends on lesion severity, structural characteristics and the proximity of PDGF and TGF-b1. Am J Pathol. 1998;152:533-546.

15. Levesque H, Girard N, Maingonnat C, Delpech A, Chauzy C, Tayot J, et al. Localization and solubilization of hyaluronan and of the hyaluronan-binding protein hyaluronectin in human normal and arteriosclerotic arterial walls. Atherosclerosis. 1994;105:51-62.

16. Papakonstantinou E, Roth M, Block LH, MirtsouFidani V, Argiriadis P, Karakiulakis K. The different distribution of hyaluronic acid in the layer of human atheromatic aortas is associated with vascular smooth muscle cell proliferation and migration. Atherosclerosis. 1998;138:79-89.

17. Wight T, Evanko S. Hyaluronan is a critical component in atherosclerosis and restenosis and in determining arterial smooth muscle cell phenotype. In: Kennedy J, Philips G, Williams P, Hascall V, eds, Hyaluronan Vol 2 Biomedical, Medical and Clinical Aspects. Cambridge Woodhead Publishing Ltd. 2002; pp.173-176.

18. Ozegowski JH, Presselt N, Härtl A, Bocker T, Sänger J, Schmidt A. Anti-atherosclerotic effect of microbial hyaluronate lyase from group B streptococci. Pharmazie. 2008;63:601-605.

19. Burgard G, Pfützner A. Intravenöse hyaluronidase therapie bei austherapierter finaler Arteriosklerose. (Intravenous hyaluronidase treatment for final stage atherosclerosis). Diabetes, Stoffwechsel und Herz. 2015:24:171-174.

20. Roberts R, Braunwald E, Muller JE, Croft C, Gold HK, Hartwell TD, et al. Effect of hyaluronidase on mortality and morbidity in patients with early peaking of plasma creatine kinase MB and non-transmural ischaemia. Br. Heart J. 1988;60:290-298.

21. Elder JB, Raferty AT, Cope V. Intra-arterial hyaluronidase in severe peripheral arterial disease. Lancet. 1980; i(8169):648-649.

22. Maroko PR, Libby P, Bloor CM, Sobil BE, Braunwald E. Reduction by hyaluronidase of myocardial necrosis following coronary artery occlusion. Circulation. 1972;46:430-437. 